\title{
The power of glove: Soft microbial fuel cell for low-power electronics
}

Jonathan Winfield, Lily D. Chambers, Andrew Stinchcombe, Jonathan Rossiter, Ioannis Ieropoulos

\begin{abstract}
A novel, soft microbial fuel cell (MFC) has been constructed using the finger-piece of a standard laboratory natural rubber latex glove. The natural rubber serves as structural and proton exchange material whilst untreated carbon veil is used for the anode. A soft, conductive, synthetic latex cathode is developed that coats the outside of the glove. This inexpensive, lightweight reactor can without any external power supply, start up and energise a power management system (PMS), which steps-up the MFC output $(0.06 \mathrm{e} 0.17 \mathrm{~V})$ to practical levels for operating electronic devices $(>3 \mathrm{~V})$. The MFC is able to operate for up to 4 days on just $2 \mathrm{~mL}$ of feedstock (synthetic tryptone yeast extract) without any cathode hydration. The MFC responds immediately to changes in fuel-type when the introduction of urine accelerates the cycling times (35 vs. $50 \mathrm{~min}$ for charge/discharge) of the MFC and PMS. Following starvation periods of up to $60 \mathrm{~h}$ at $0 \mathrm{mV}$ the MFC is able to cold start the PMS simply with the addition of $2 \mathrm{~mL}$ fresh feedstock. These findings demonstrate that cheap MFCs can be developed as sole power sources and in conjunction with advancements in ultra-low power electronics, can practically operate small electrical devices.
\end{abstract}

\section{Keywords:}

Microbial fuel cell, Natural rubber, Power management system, Urine, Conductive latex Energy harvesting

\section{Introduction}

The first report of microbial fuel cells (MFCs) was over 100 years ago when M.C. Potter discovered that 'the disintegration of organic compounds by micro-organisms was accompanied by the liberation of electrical energy' [1]. In subsequent years, power output from MFCs has increased many-fold but to date individual reactors cannot produce enough energy to directly power the majority of commercial electrical devices [2]. One method for amplifying output is to connect multiple reactors together electrically. In this way a small robot (EcoBot I) was operated by employing eight $25 \mathrm{~mL}$ MFCs that charged a bank of six 
capacitors designed to discharge when a particular threshold was reached [3]. For larger applications, the stacking of MFCs is needed to produce the amounts of power required even for a pulsated locomotion [4]. However, stacking brings with it complications such as cell reversal [5] because the system is limited by the underperforming cell within the stack [6], an occurrence that can be exaggerated by the biological nature of MFCs. Furthermore, stacking involves a design complexity, which may not be required for small-scale implementations. In combination with MFC developments, significant advances have been made in low power electronics. DC/DC converters are now able to boost voltage from relatively low starting levels, but unfortunately most individual MFCs are still not able to directly drive them [7]. This is because most off-the-shelf DC/DC converters consume more power than is produced by as many as 10 miniature MFCs [8]. In order to harvest the useful energy from a MFC, power management systems (PMS) are needed which can interface the reactor with the load by initially accumulating electrical energy and subsequently driving the load intermittently [7]. For this to be practical the MFC must first be able to produce a voltage in excess of the minimum input voltage of the PMS. This, especially in a fluctuating or heterogeneous environment, could be difficult to maintain. Over the years there has been a significant reduction in the input voltage required to operate these electronic systems. Shantaram et al. (2005) [9] ran a system using a minimum 0.9 V from their large reactor. Meehan et al. (2011) [10] designed a system that required a minimum input voltage of $0.6 \mathrm{~V}$. The PMS developed by $\mathrm{Wu}$ et al. (2012) [8] was able to operate with an input voltage in the region of $0.2 \mathrm{e} 0.4 \mathrm{~V}$ from their stack of miniature MFCs. The lowest input voltage to date (to the authors knowledge) that was successfully able to operate a PMS is $0.18 \mathrm{~V}$ [11]. There is therefore a continuing requirement to find ultra-low power voltage electronics that could preferably be powered by individual MFCs, as well as an on-going effort to develop bespoke energy harvesting systems (EcoBots). Despite the aforementioned progress in MFC technology over recent years, unsustainable and expensive materials are still being employed, making the technology difficult to implement or scale-up. Platinum (as used in the first MFC by Potter, $1911[1])$ is still regularly incorporated into the cathode as a catalyst and unsustainable chemicals such as ferricyanide are still employed to boost power. Commercially manufactured proton exchange membranes (PEM) are commonplace though their operation is sub-optimal in the ambient conditions suitable for the survival of anodic microorganisms [12]. When attempting to avoid materials such as platinum, researchers can encounter other challenges, such as the requirement for frequent hydration, when it comes to un-treated carbon cloth cathodes. [13]. These introduce unwanted complexity into the design and 
maintenance of the system (as demonstrated by the EcoBot-III robot [14]). Hydration requirements of the cathode could in fact negate the benefit of the removal of platinum in the first place. It is therefore imperative to consider materials that not only decrease cost but also contribute to low maintenance and simplified operation. Furthermore, when designing MFCs for operation in remote locations it would be desirable to use inexpensive, biocompatible and/or biodegradable materials. The main aims of the current study were therefore (i) to design and build an inexpensive biodegradable MFC reactor (ii) to run ultra-low power electronics using the aforementioned MFC (iii) to demonstrate that the system is capable of self-starting without the need for an external power supply and (iv) to investigate the response from different feedstocks. To achieve these objectives, MFCs were constructed using natural rubber from the finger pieces of laboratory gloves. The soft glove performed the dual function of structural material as well as proton exchange membrane. An inexpensive flexible, conductive, synthetic rubber cathode was developed that was tested in terms of stability and hydration requirements. A low operating voltage off-the-shelf energy harvesting board (Advanced Linear Devices Inc.) was tested using the electricity generated from the glove MFC. To demonstrate the immediate response to different feedstocks, a synthetic medium and real urine were compared.

\section{Experimental}

\subsection{MFC construction and operation}
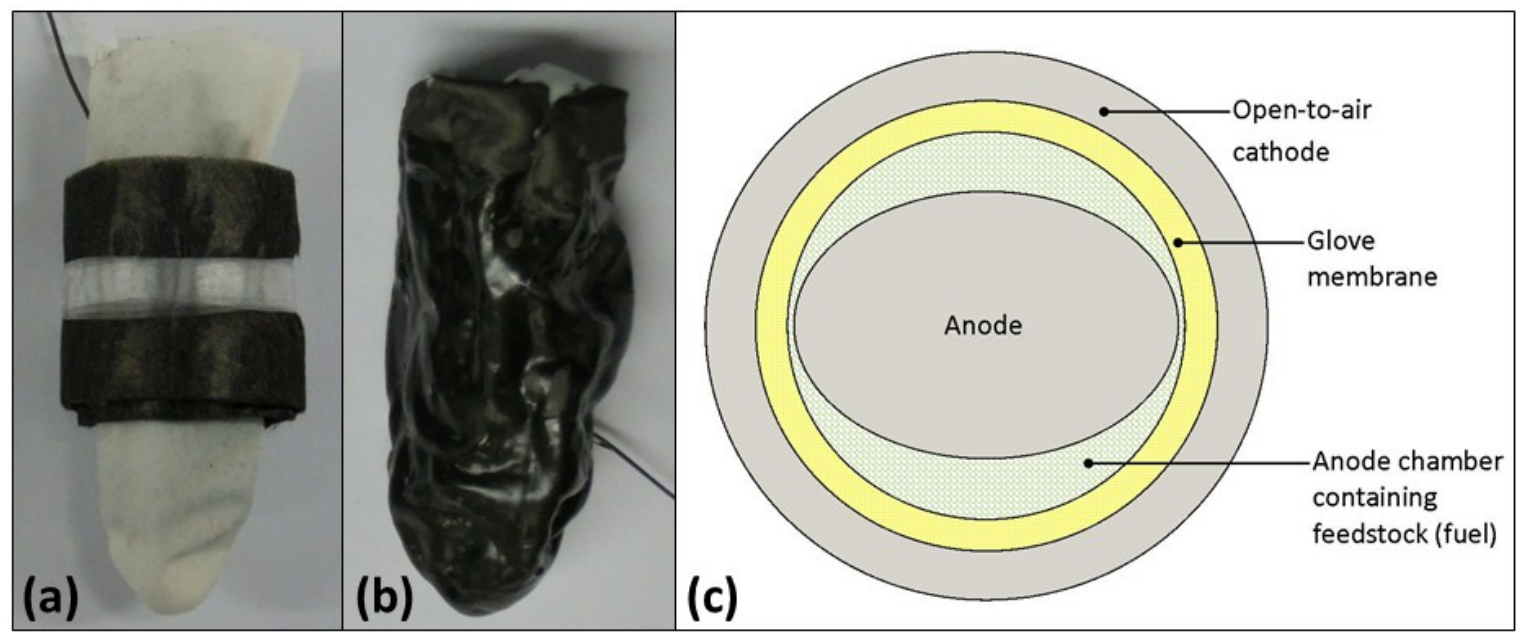

Fig. 1. Natural rubber latex glove microbial fuel cell; (a) carbon veil cathode bound with parafilm at the centre; (b) conductive latex cathode and (c) schematic cross section (topside view).

The finger pieces were cut from laboratory natural rubber latex gloves (SemperGuard powder-free, size 8-9), and had an empty chamber volume of $7 \mathrm{~mL}$. For the anode a $180 \mathrm{~cm}^{2}$ 
$(9 \times 20 \mathrm{~cm})$ piece of carbon veil was folded into a cylindrical shape (projected surface area of approximately $19 \mathrm{~cm} 2$ ) and a piece of titanium wire was threaded all the way through as a current collector. Two cathode types were tested with the glove finger membranes; carbon veil as control (CCV-MFC [Fig. 1a]) and polyurethane-based conductive latex (CL-MFC [Fig. 1b]); a cross-section schematic of the glove MFCs is shown in Fig. 1c. The carbon veil cathodes were prepared by folding and wrapping $180 \mathrm{~cm} 2$ of untreated carbon fibre veil around the outside of the glove finger. Parafilm was used to hold the material securely and titanium wire was threaded through and employed as a current collector. The conductive latex cathode was prepared as follows: synthetic, polyurethane rubber coating (Plasti-Dip, Petersfield, UK) was mixed with naphtha before graphite powder (Fisher Chemical, Loughborough, UK) was blended in. The wet weight ratio of rubber coating without naphtha to graphite powder was 2:3. A piece of titanium wire was adhered to the latex surface using wire glue (Anders Products, MA, USA) to act as current collector. The conductive latex was painted over the outside of the natural rubber glove finger and wire. Once the cathode conductive latex had cured, the anode was inserted into the coated glove finger MFC. Four MFCs (two of each cathode type) were inoculated using primary sludge (Wessex Water, Saltford, UK) enriched with $1 \%$ tryptone and $0.5 \%$ yeast extract. Following inoculation, the MFCs were batch-fed with sterile TYE ( $1 \%$ tryptone, $0.5 \%$ yeast extract in dionised water) for 6 months. Feeding involved topping up with 1-2 mL every few days which was essentially compensating for the consumption and/or evaporation losses. In the early stages of the experiments, when conductive latex was being compared to carbon veil cathodes, the cathodes were periodically hydrated with tap water. Resistive loads of $2 \mathrm{k} \Omega$ value were used during inoculation and subsequent operation. After 6 months operation the best performing conductive latex MFC (CL-MFC) was selected to operate the power management system (PMS). In order to acclimatise the chosen MFC to the requirements of the PMS, the fixed external load was changed from $2 \mathrm{k} \Omega$ to $1 \mathrm{k} \Omega$ for two weeks prior to connection to the energy harvesting board. When connected to the PMS the CL-MFC was batch-fed TYE every few days for two months before the feedstock was changed to fresh urine. Urine samples were provided by healthy volunteers on a normal diet with no history of urinary tract or renal disease. All experiments were performed at ambient temperature conditions $\left(22 \pm 1^{\circ} \mathrm{C}\right)$.

\subsection{Power management system}




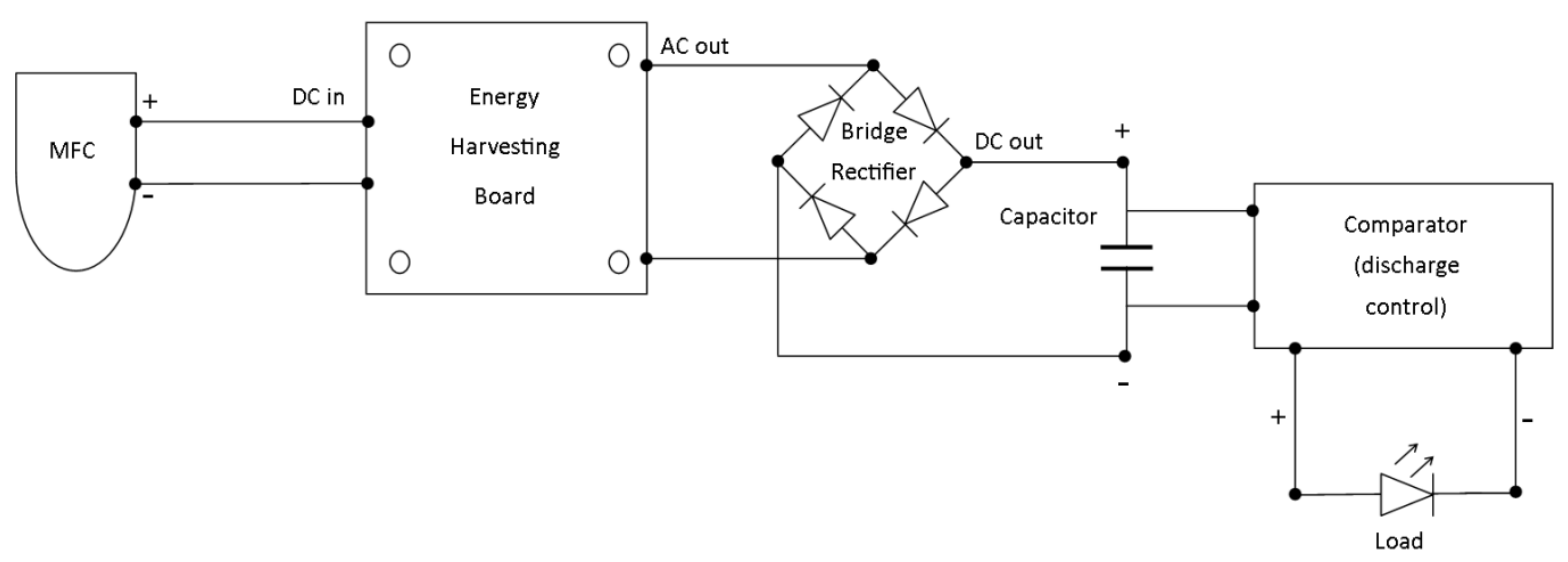

Fig. 2. Schematic of the power management system including MFC, energy harvesting board and comparator discharge board

The CL-MFC was connected to a power management system (PMS) consisting of an inductor-based energy harvesting board (EH4295, Advanced Linear Devices Inc., CA, USA), a bridge rectifier (BAT43 small signal diode, RS Components Ltd, Corby, UK), a capacitor $(6.3 \mathrm{mF})$ and a custom made comparator board with added hysteresis (Fig. 2). The comparator limited the capacitor voltage to $3.1 \mathrm{~V}$ and discharged to a minimum voltage of 2 V. A variety of real applications were employed as loads during the course of experimentation including an electric motor-driven windmill, an LED and an ionic polymer metal composite (IPMC) artificial muscle.

\subsection{Data logging}

During the initial months of operation all data were recorded using an ADC-16 Channel Data Logger (Pico Technology Ltd., Cambridgeshire, UK). When the MFC was connected to the energy harvesting board, an Agilent LXI (34972A) data acquisition/switch unit was employed to monitor the synchronised behaviour of both the MFC and capacitor. Recorded data were processed and analysed using the GraphPad Prism version 6 software package (GraphPad, San Diego, California, USA). Current (I) in amperes (A) was calculated using Ohm's law, $\mathrm{I} 1 \frac{1}{4} \mathrm{~V} / \mathrm{R}$, where $\mathrm{V}$ is the measured voltage in volts $(\mathrm{V})$ and $\mathrm{R}$ is the known value of the external load resistor in ohms $(\Omega)$. Power $(\mathrm{P})$ in watts $(\mathrm{W})$ was calculated by multiplying voltage with current: $\mathrm{P}=\mathrm{I} \times \mathrm{V}$.

\subsection{Bi-directional polarisation sweeps}


Bi-directional polarisation experiments were performed using an automated computercontrolled variable resistor system [15]. This apparatus swept 60 resistance values starting from $1 \mathrm{M} \Omega$ down to $3 \Omega$ at a sample rate of $5 \mathrm{~min}$, before sweeping back up (at the same time constant) to $1 \mathrm{M} \Omega$. There was no 'rest period' between sweeps and so effectively the MFCs were held at $3 \Omega$ for $10 \mathrm{~min}$ ( $5 \mathrm{~min}$ forward, $5 \mathrm{~min}$ reverse).

\section{Results and discussion}

\subsection{Carbon veil vs. conductive latex cathodes}

In order for a MFC to power useful devices and/or operate in remote locations it should ideally be inexpensive, able to provide a stable and reliable performance, and require minimal maintenance [3]. One method of cutting costs is to produce cathodes without platinum; however, the use of electrode materials without this expensive catalyst often require cathode hydration in order to maintain a moist environment for the transport of ionic species and oxygen reduction [16]. This provides the added cost and complexity of incorporating the hydration process into operation. In addition, catholyte make-up and flow-rate have a direct effect on voltage output [17] and so the sensitivity of a device designed for sensing a target analyte could be detrimentally affected. Alternative cathode materials are being developed that avoid the need for hydration, but preparation of these often requires specific apparatus and lengthy process times. The current study developed a novel conductive latex cathode requiring short preparation times without the need for any specialised equipment or materials.
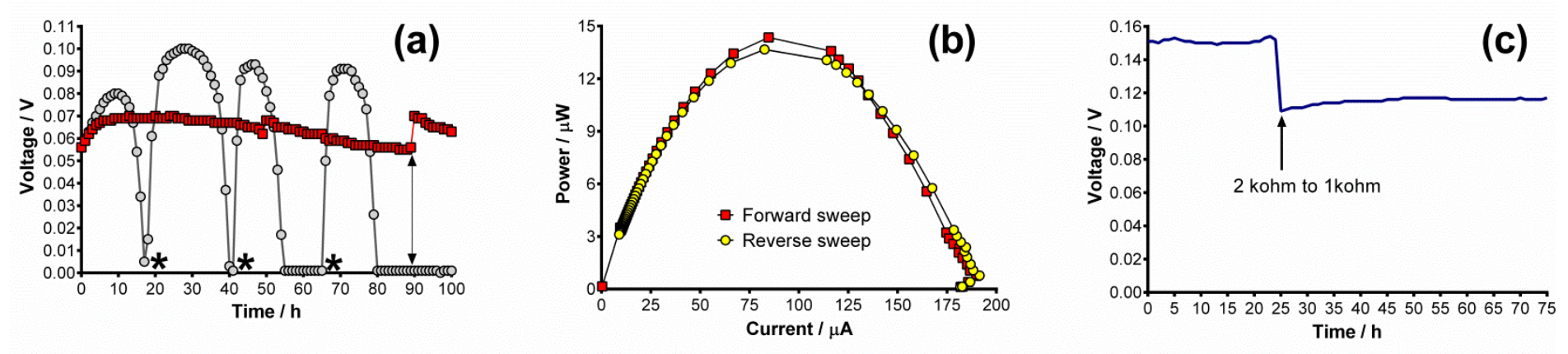

Fig. 3. Performance of glove MFCs: (a) after 1 week temporal data comparing MFC with conductive latex cathode (squares) vs carbon veil cathode (circles), asterisks indicate cathode hydration, arrow indicates feeding of TYE to anodes; (b) bi-directional power curves produced from MFC with conductive latex cathode (CL-MFC) after 6 months; (c) steady-state voltage of CL-MFC after 6 months showing the point when $R_{E X T}$ changed from 2 $\mathrm{k} \Omega$ to $1 \mathrm{k} \Omega$.

During the early stages of operation the MFCs with conductive latex cathode (CL-MFCs) were not as efficient in terms of power production when compared to the control carbon veil 
cathodes (CCV-MFC). However, as illustrated in Fig. 3a which shows the best performing MFC of each type during the second week of operation, the CL-MFC, despite producing an inferior output under the $2 \mathrm{k} \Omega$ load, did not require hydration of the cathode. The CCV-MFCs on the other hand did rely on hydration, since the MFC's output rapidly declined to $0 \mathrm{mV}$ as the cathode dehydrated; full recovery was recorded once water was added (as indicated by the asterisks). Interestingly, the CL-MFCs showed no response to hydration. The carbon veil's reliance on hydration was more marked when the MFCs were fed with anolyte (but not hydrated) as indicated by the arrow (at $90 \mathrm{~h}$ on Fig. 3a). Here the CL-MFCs showed improvement following the addition of fresh fuel, which is in contrast to the CCV-MFCs that no response due to the non-functioning (dehydrated) cathode. Because of these lower maintenance requirements, the best performing CL-MFC was selected as the reactor of choice for energising the power management system and all subsequent data are based on the MFC with conductive latex cathode. In addition to minimum maintenance the stability of the CL-MFC was verified through bi-directional polarisation sweeps. Fig. $3 \mathrm{~b}$ shows that there Fig. 1. Natural rubber latex glove microbial fuel cell; (a) carbon veil cathode bound with parafilm at the centre; (b) conductive latex cathode and (c) schematic cross section (topside view) was little hysteresis between forward and reverse sweeps, which is not the case with commercially available PEMs that often demonstrate significant loss in power during the reverse sweep $[5,18,19]$. As previously reported [20], MFCs employing natural rubber as membrane improve over time as the material degrades, and after 6 months the CL-MFC continued to improve whilst maintaining a steady performance. Fig. $3 \mathrm{c}$ shows that under a 2 $\mathrm{k} \Omega$ load the MFC had more than doubled the voltage output compared to the first month of operation (Fig. 3a) and when the load was decreased to $1 \mathrm{k} \Omega$ it continued to operate in a stable manner. The energy harvesting board requires a start-up voltage of $60 \mathrm{mV}$ at an internal resistance of $950 \Omega$ according to the manufacturer's guidelines and so the CL-MFC performance $(110 \mathrm{mV})$ under the $1 \mathrm{k} \Omega$ was considered capable of operating the PMS. The lightweight materials of the individual CL-MFC (Fig. 1b) could present an additional advantage over other MFC designs should total on-board weight be an essential requirement of the end application (e.g. a mobile robot). The empty chamber volume of each CL-MFC was approximately $7 \mathrm{~mL}$ and once the anode was inserted and wetted the amount of feedstock required for each new feed was $2 \mathrm{~mL}$. These parameters are comparable to the MFCs used on the recent EcoBot-III robot, which comprised 48 MFCs [14]. The total dry weight of the CL-MFC ( $<2 \mathrm{~g})$ is significantly lower compared to the individual EcoBot MFCs (25 g) that were constructed from Nanocure photopolymer. Although durability and 
robustness will be essential in some environments, a lightweight compliant device would improve the total energy efficiency and could be employed in scenarios that would not be feasible for bulky, rigid machines. Furthermore the combined cost of materials required to build each CL-MFC was calculated at less than 10 UK pence (or $<15$ US cents) making the reactors very cost effective.

\subsection{Operating the power management system (PMS)}
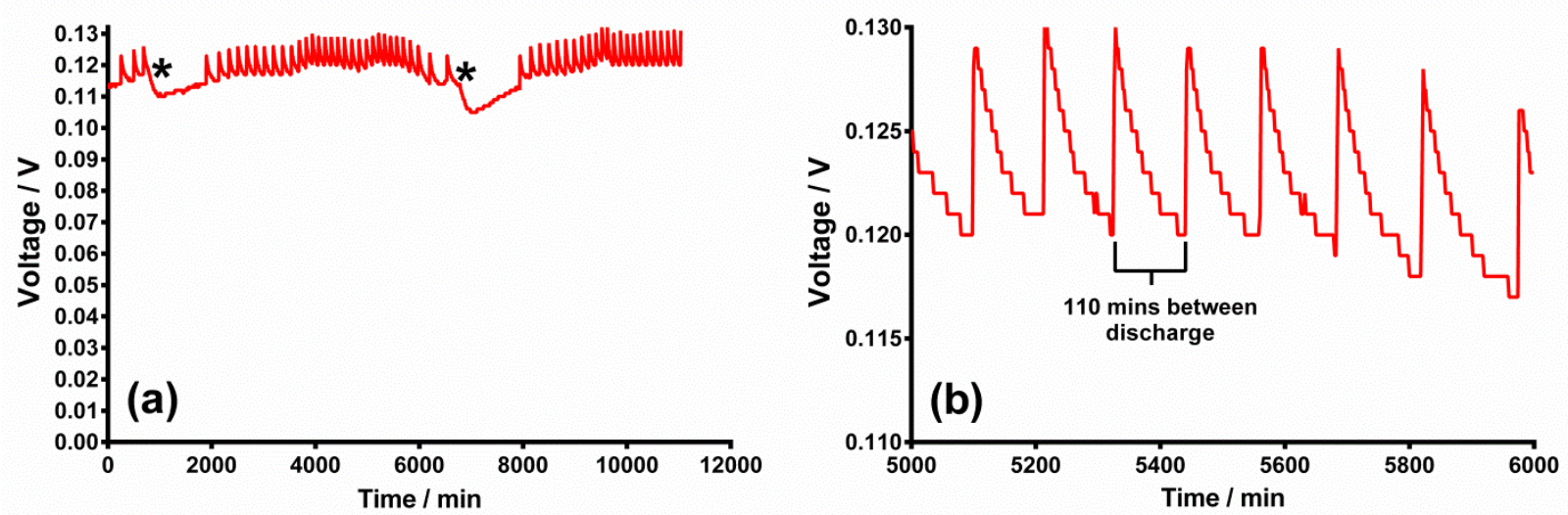

Fig. 4. MFC behaviour when connected to the energy harvesting board after one week of operation; (a) shows two points where MFC was fed $2 \mathrm{~mL}$ of TYE as indicated by asterisks; (b) higher resolution image of the fastest charge/discharge cycle.

To initially test the PMS, the CL-MFC was connected to the energy harvesting board without being connected to the comparator board, and in $4 \mathrm{~h}$ it was able to charge the capacitor from 0 to $5 \mathrm{~V}$. The capacitor was manually discharged and the circuit was then set up to include the comparator board, which initiates the capacitor discharge from $3.1 \mathrm{~V}$ down to $2 \mathrm{~V}$. This is the energy used for periodically operating (or firing) the electrical device and at the $2 \mathrm{~V}$ point, the capacitor begins to charge again. Fig. 4 shows the behaviour of the MFC after 1 week of being connected to the PMS. Each peak and trough represent one charge/discharge cycle where the MFC voltage decreases gradually as the capacitor charge increases. When the capacitor begins to discharge at $3.1 \mathrm{~V}$ the MFC immediately rebounds back to the higher voltage levels. The MFC behaviour during each charge/discharge cycle is determined by the energy harvesting board and fixed-resistance-experiments were carried out to determine the ohmic range between each peak (i.e. capacitor charging) and trough (i.e. directly prior to the capacitor discharge). The range was calculated as approximately $100 \Omega$ (data not shown), with the lowest voltage occurring at $950 \Omega$ and the highest at $1050 \Omega$. The asterisks in Fig. $4 \mathrm{a}$ indicate the points at which the anode was fed $2 \mathrm{~mL}$ of TYE feedstock. Following the introduction of fresh medium the MFC voltage initially declines before rising and once again 
initiating capacitor discharge. This initial decline, when fed sterile TYE, is likely due to the time required to breakdown the complex molecules into more accessible nutrients for digestion by the electro-active community. Furthermore, analysis of TYE as a feedstock in other experiments revealed that the conductivity increased many-fold after several hours in an MFC, a phenomenon that would considerably lower the internal resistance over time [21], but not necessarily during the initial stages of feeding. There were 31 discharges between the two feeds with the fastest charge/discharge cycle lasting $110 \mathrm{~min}$ as highlighted in the magnified area in Fig. 4b. The CL-MFC was able to continuously charge/discharge and operate for up to 4 days on just $2 \mathrm{~mL}$ of feedstock and as previously stated, the conductive latex cathode required no hydration. At the end of the feeding cycle the MFC dropped to voltages too low to support the energy demands of the PMS.

\subsection{Synthetic media (TYE) versus urine}
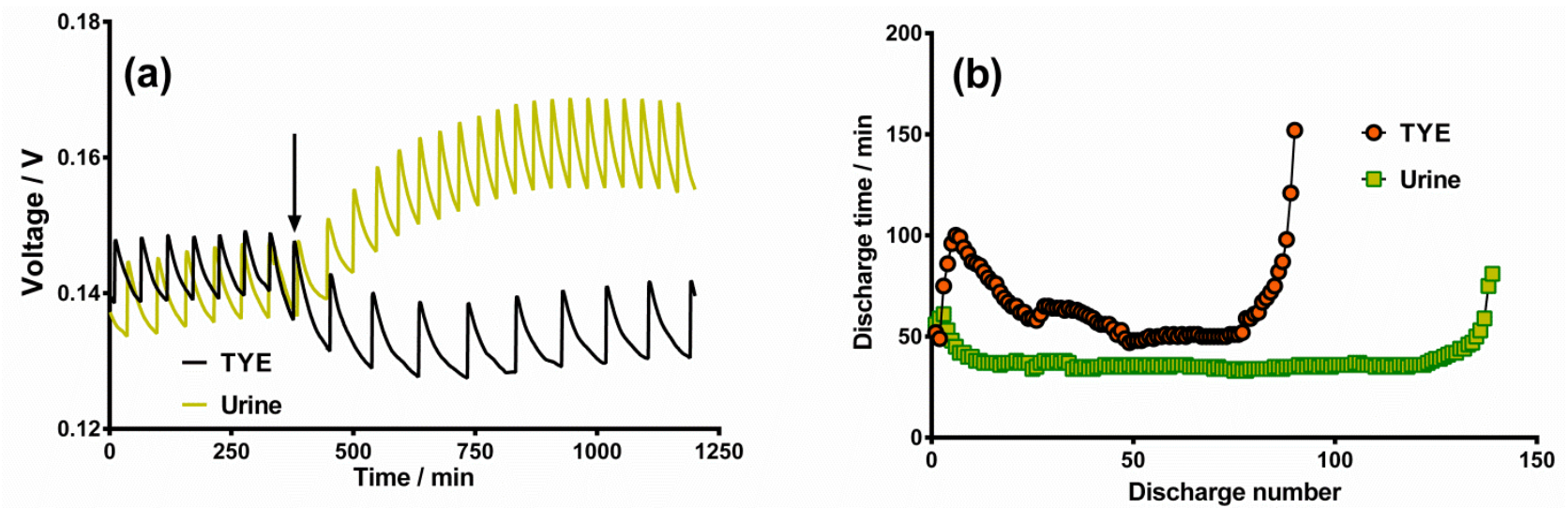

Fig. 5. Comparison between a synthetic medium (TYE) and urine as fuels; (a) MFC response to being fed either urine or TYE, arrow indicates the point when the feedstock was introduced; (b) the number and duration of discharges from initial $1 \mathrm{~mL}$ feed to depletion.

The previous section demonstrated that a single MFC was able to power an electronic device through the power management system using a synthetic medium as substrate. In order to test the sensitivity and versatility of the system, urine was fed to the CLMFC. Urine was used because it has recently been shown to be an ideal fuel for electricity production in MFCs [22]. Fig. 5a shows the behaviour of the same MFC in response to being fed just $1 \mathrm{~mL}$ of either TYE or urine. The data are plotted on the same axes in order to directly compare the responses to different fuels. Prior to the arrow (i.e. before being topped up with either TYE or urine) the MFC had been fed TYE. When the MFC is topped up with $1 \mathrm{~mL}$ of fresh TYE, as previously discussed, there is an initial decline in voltage and an increase in the time taken to charge/discharge. When the MFC is topped up with $1 \mathrm{~mL}$ of urine there is an immediate 
increase in power and a subsequent reduction in time for each charge/discharge cycle. This is supported in Fig. 5b, which shows the number and frequency of discharges over two feeding cycles (one with TYE and the other with urine) running from initial feeding to depletion of feedstock and performed during the second month connected to the PMS. The TYE cycle displays an initial increase in time between charge/discharge before the frequency eventually becomes stable at approximately 50 min per charge cycle, which is a considerable improvement from the first week (Fig. 4b). As the feedstock becomes depleted the discharge frequency gradually decreases until the last discharge is achieved in $150 \mathrm{~min}$, after which the CL-MFC does not produce enough power to maintain the PMS and both reactor and capacitor decline to zero voltage as will be discussed further in Section 3.4. The urine cycle is relatively stable throughout with an average charge cycle of approximately $35 \mathrm{~min}$, which eventually slows as the anolyte runs out and the final discharge takes $81 \mathrm{~min}$ before the system shuts down. The MFC managed 90 discharges (firings of the electronic system) when fed $1 \mathrm{~mL}$ of TYE compared to 138 discharges when fed $1 \mathrm{~mL}$ urine. These results confirm recent findings [22] that a natural and ubiquitous substance such as urine can be employed as a fuel for electronic devices and/or biosensors.

\subsection{System start-up}

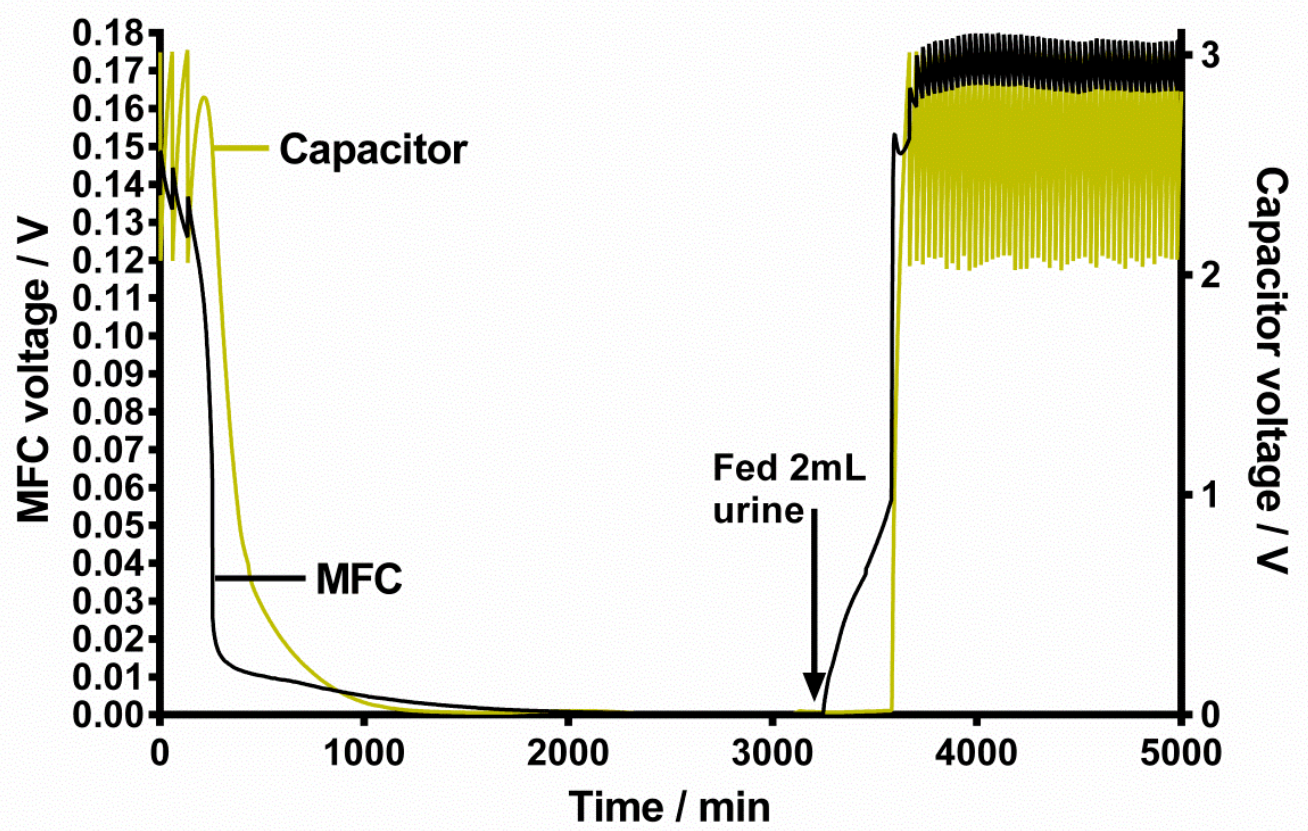

Fig. 6. System shut-down and subsequent recovery (cold start) when MFC fed $2 \mathrm{~mL}$ of urine. 
For a MFC that is operating as a biosensor itself, is powering a biosensor or is powering any device in a remote location, it would be advantageous if it could restart (a.k.a. cold start) the system should shut-down occur due to anolyte depletion. This would be particularly valuable if such a system is required to operate only under certain conditions, for example in the presence of a particular compound or analyte. The CL-MFC proved to be capable not only of self-recovery following desiccation but was also able to restart the electronic energy harvester without any external power supply after periods of up to $60 \mathrm{~h}$. Fig. 6 shows the gradual drop in voltage of both CL-MFC and capacitor following anolyte depletion. After approximately $2000 \mathrm{~min}$, both had declined to $0 \mathrm{mV}$ as the PMS had continued to draw more power than was being supplied. After $3250 \mathrm{~min}, 2 \mathrm{~mL}$ urine was fed to the MFC and an immediate response was observed as the MFC voltage began to increase. During the first few hours of MFC recovery, the capacitor remained at zero Volts until the MFC reached $57 \mathrm{mV}$, which was enough to switch on the energy harvesting board and restart the whole system. At this point the voltage of the CL-MFC and capacitor rose steeply and the cycle of charge/discharge was restarted. The system was not left inactive for periods longer than $60 \mathrm{~h}$ and so potentially a restart could take place after longer phases of hibernation which is an area for future work. The ability to self-start is a powerful tool for an autonomous device, because fuel availability may vary in the environment. Providing there is a continuous supply of feedstock, the system could carry on indefinitely and the glove MFC reported herewith has been operational without a decline in power output for more than 1 year. This is despite some physical deterioration in the form of small cracks in the natural rubber at the lip of the MFC. Because the natural rubber is prone to biodegradation the MFC will have an 'expiry date' but this can be another beneficial trait if recovery of the remote system is infeasible. Clearly the electronics used in the current study are not biodegradable and thus further research is needed before whole biodegradable biosensors and robots may be developed. The energy harvesting board was only able to provide a power efficiency of approximately $45 \%$ according to the manufacturer's guidelines, but it is envisaged that as ultralow power electronics evolve, power efficiency will increase which can only help to accelerate the scale-up of microbial fuel cells.

\section{Conclusions}


A soft, lightweight and inexpensive MFC was developed using laboratory gloves. A novel conductive latex was prepared that performed the role of cathode without the need for hydration. By employing an ultra-low voltage energy harvesting board, the voltage from an individual glove MFC (0.06-0.17 V) was converted into practical voltage levels ( $>3 \mathrm{~V})$ able to power small standalone practical applications periodically. An external power source was not required and the system could self-start following complete shutdown simply with the addition of $2 \mathrm{~mL}$ of feedstock. Increased firings at shorter intervals were produced when urine was introduced as feedstock compared to synthetic TYE.

\section{Acknowledgements}

The authors would like to thank The Leverhulme Trust for their financial support through Project Grant RPG-362. The Bill \& Melinda Gates Foundation (GCE: OPP1044458) supported parts of the urine work. Ioannis Ieropoulos is an EPSRC Career Acceleration Fellow (EP/I004653/1).

\section{References}

[1] M.C. Potter, Proc. R. Soc. Lond. B 84 (1911) 260-276.

[2] H. Wang, Z. Ren, J. Park, J. Power Sources 220 (2012) 89-94.

[3] I. Ieropoulos, J. Greenman, C. Melhuish, in: Proceedings of the 2nd International Symposium on Imitation of Animals and Artifacts, 2003, pp. 191-194.

[4] I. Ieropoulos, J. Greenman, C. Melhuish, Int. J. Energy Res. 32 (2008) 12281240.

[5] P. Aelterman, K. Rabaey, H.T. Pham, N. Boon, W. Verstraete, Environ. Sci. Technol. 40 (2006) 3388-3394.

[6] S.E. Oh, B.E. Logan, J. Power Sources 167 (2007) 11-17.

[7] D. Zhang, F. Yang, T. Shimotori, K. Wang, Y. Huang, J. Power Sources 217 (2012) 65-71.

[8] P.K. Wu, J.C. Biffinger, L.A. Fitzgerald, B.R. Ringeisen, Process. Biochem. 47 (2012) 1620-1626.

[9] A. Shantaram, H. Beyenal, R. Raajan, A. Veluchamy, Z. Lewandowski, Environ. Sci. Technol. 39 (2005) 5037-5042.

[10] A. Meehan, H. Gao, Z. Lewandowski, IEEE Trans. Power Electron. 26 (2011) 176-181.

[11] F. Yang, D. Zhang, T. Shimotori, K. Wang, Y. Huang, J. Power Sources 205 
(2012) 86-92.

[12] J.C. Biffinger, R. Ray, B. Little, B.R. Ringeisen, Environ. Sci. Technol. 41 (2007) 1444-1449.

[13] A. Montebelli, R. Lowe, I. Ieropoulos, C. Melhuish, J. Greenman, T. Ziemke, Artificial Life XII, MIT Press, 2010, pp. 749-756.

[14] I. Ieropoulos, J. Greenman, C. Melhuish, I. Horsfield, Artificial Life XII, MIT Press, 2010, pp. 733-740.

[15] N. Degrenne, F. Buret, B. Allard, P. Bevilacqua, J. Power Sources 205 (2012) 188-193.

[16] J.R. Kim, G.C. Premier, F.R. Hawkes, R.M. Dinsdale, A.J. Guwy, J. Power Sources 187 (2009) 393-399.

[17] I. Ieropoulos, C. Melhuish, J. Greenman, Bioinspiration Biomimetics 2 (2007) S83-S93.

[18] N. Degrenne, P. Ledezma, P. Bevilacqua, F. Buret, B. Allard, J. Greenman, I. Ieropoulos, Bioresour. Technol. 128 (2013) 769-773.

[19] J. Winfield, L. Chambers, J. Rossiter, I. Ieropoulos, Bioresour. Technol. 148 (2013) 480-486.

[20] J. Winfield, I. Ieropoulos, J. Rossiter, D. Greenman, J. Patton, Biodegradation 24 (2013) 733-739.

[21] L. Huang, B.E. Logan, Appl. Microbiol. Biotechnol. 80 (2008) 349-355.

[22] I. Ieropoulos, J. Greenman, C. Melhuish, Phys. Chem. Chem. Phys. 14 (2012) 94-98. 